\title{
Ethnobotanical Study of Medicinal Plants on Arthritis Used by Chaoshan in Guangdong, China
}

\author{
Peihong Chen ${ }^{1}$, Fuchun Zheng ${ }^{2}$, Yanmei Zhang ${ }^{1}$, Fenfei Gao1, Yicun Chen ${ }^{1,3 *}$ and Ganggang Shi ${ }^{1,3 *}$ \\ ${ }^{1}$ Department of Pharmacology, Shantou University Medical College, Shantou 515031, China \\ ${ }^{2}$ Department of Pharmacy, First Affiliated Hospital, Shantou University Medical College, Shantou 515031, China \\ ${ }^{3}$ Traditional Chinese Medicine Laboratory, Shantou University Medical College, Shantou 515031, China
}

\begin{abstract}
Ethnopharmacological relevance: An ethnobotanical survey was conducted to collect information of medicinal plants on Arthritis relating to anti-inflammatory and Analgesia effect by Chaoshan-Shantou People living in Guangdong.
\end{abstract}

Aim of the study: This investigation was to document valuable knowledge represented as Chaoshan herbal medicine.

Materials and methods: Information was obtained from interviews and by reviewing studies of Chaoshan and Cantonese reported in the literature.

Results: Our data covered 86 species belonging to 82 genera in 52 families. In the search of the PubMed database, there are 28 herbs that have been studied, which have the most anti-inflammatory effects of the herb, followed by analgesia.

Conclusions: Due to the rapid disappearance of urbanization and industrialization of traditional culture and natural resources, indicating that the recorded information may be lost forever. Therefore, there is an urgent need to record the value of Chaoshan medicinal knowledge and encourage the transfer to the next generation.


Keywords: Chaoshan herbal medicine; Arthritis; Anti-inflammation; Analgesia

\section{Introduction}

The chronic and acute inflammation can lead to serious organs and tissues damage. Arthritis refers to occur in the human body joint and surrounding tissue inflammatory diseases, points to dozens. There are more than 100 million Chinese patients with arthritis and increasing in number. Its clinical manifestations are red, swollen, hot, painful, functional disability and joint deformities, which lead to joint disability, affecting the quality of life of patients. The etiology of arthritis is complex, mainly related to inflammation, autoimmune reaction, infection, metabolic disorders, trauma, degenerative diseases and so on. According to the pathogenesis and clinical manifestations, arthritis can be divided into rheumatoid arthritis, rheumatoid arthritis, osteoarthritis, gout arthritis and ankylosing spondylitis, etc.

Non-steroidal anti-inflammatory drugs (non-steroidal antiinflammatory drugs) are the most commonly used drugs for the treatment of pain caused by inflammatory and degenerative diseases, including reactive arthritis [1-4]. The main mechanism is considered to be the two isoforms of epoxy synthase blockade, namely COX-1 and

*Corresponding authors: Yicun Chen, Department of Pharmacology, Shantou University Medical College, Shantou 515031, China, Tel: +8675488900432; E-mail: chenyicun@yeah.net

Ganggang Shi, Traditional Chinese Medicine Laboratory, Shantou University Medical College, Shantou 515031, China, Tel: +8675488900430; E-mail: ggshi@stu.edu.cn

Received December 13, 2016; Accepted December 20, 2016; Published December 24, 2016

Citation: Chen P, Zheng F, Zhang Y, Gao F, Chen Y, et al. (2016) Ethnobotanical Study of Medicinal Plants on Arthritis Used by Chaoshan in Guangdong, China. Med Chem (Los Angeles) 6: 715-723. doi: 10.4202/2161-0444.1000420

Copyright: $\odot 2016$ Chen $\mathrm{P}$, et al. This is an open-access article distributed under the terms of the Creative Commons Attribution License, which permits unrestricted use, distribution, and reproduction in any medium, provided the original author and source are credited. 
Citation: Chen P, Zheng F, Zhang Y, Gao F, Chen Y, et al. (2016) Ethnobotanical Study of Medicinal Plants on Arthritis Used by Chaoshan in Guangdong, China. Med Chem (Los Angeles) 6: 715-723. doi: 10.4172/2161-0444.1000420

COX-2 expression, resulting in a decrease of prostaglandin synthesis. In fact, the therapeutic effect of non-steroidal anti-inflammatory drugs is due to its inhibition of the COX-2 isoform, in inflamed tissue inducing capacity, and some of the most common side effects of their is, generally with homeostasis and inhibition of COX-1 isoform.

Similarly, tumour necrosis factor alpha (TNF-alpha) in the pathogenesis of rheumatoid arthritis (RA) plays a core role, because it is in arthritis inflammatory and destructive processes vertices. Using anti TNF alpha blocking clinical trials showed, TNF regulation of inflammatory cytokine and chemokines, adhesion molecule expression, resulting in joint leukocyte migration, matrix metalloproteinases (MMPs), and joint destruction, and vascular endothelial growth factor (VEGF) and angiogenesis [5-9]. TNF-alpha gene expression in complex control, and p38 mitogen activated protein kinase pathway controlling translation possible actions on the 3-untranslatedregion [10], and with the 5-untranslated/promoter region contains multiple transcription factor binding sites, including nuclear factor B (NF- $\mathrm{BB}$ ) and activator protein-1 and nuclear factor of interleukin IL - 6 (IL-6) gene, and activation of $\mathrm{T}$ cells [11-13]. NF- $\kappa \mathrm{B}$ has recently attracted particular attention because of its ability to regulate macrophage

TNFa production induced in response to lipopolysaccharide (LPS), ultraviolet light, phorbol myristate acetate, or contact with cytokineactivated $\mathrm{T}$ cells $[14,15]$.

Chaoshan area is located in the eastern part of Guangdong Province. Complex geographical factors as well as mountains, sea, plain both superior natural conditions, making medicinal plant resources in the eastern region is very rich. Most of them are used based as antiinflammatory and analgesia herbs. There is an urgent need to record the value of Chaoshan medicinal on anti-inflammation and analgesia and encourage the transfer to the next generation (TOC Graphic).

\section{Materials and Methods}

\section{Study area}

Chaoshan area is located in the eastern part of Guangdong Province, is located approximately east longitude $114^{\prime} 53 \sim 117^{\prime} 16^{\prime}$, north latitude $22 ' 31$ ' 24'15', The Tropic of cancer through this place. The central region including Shantou, Chaozhou, Jieyang, Shanwei City, a total area of 157720 square kilometers. At the junction of the northeast and Fujian Province, the northwest bordering near Xinghai area, southeast region near the South China Sea. The northeast, northwest mountains, a natural barrier between regions, the main peak of Lantau Peak Phoenix bird bun 1498 meters above sea level, the highest peak in the territory, while the southeast coast is flat, rivers of Chaoshan Plain, west coast is a multistage platform. The climate in this region is a subtropical climate throughout the year, affected by the monsoon, but the coastal and inland areas are slightly different, plenty of sunshine, the annual average temperature is 21 to 22 degrees Celsius, rainfall, the average annual rainfall is 1300 to $2200 \mathrm{~mm}$, relative humidity above $80 \%$ soil. Complex and varied types of soil, lateritic red soil, followed by yellow soil, red soil, alluvial, paddy soil, saline soil. Complex geographical factors as well as mountains, sea, plain both superior natural conditions, making plant resources in the eastern region is very rich. According to preliminary statistics there are medicinal plants 228 families, 903 genera, 1599, 73 variants [16].

\section{Ethnobotanical survey}

For this study, ethnopharmarcologists, pharmacognosists and botanists, translators with medicinal background from the Shantou University Medical college and local people with medicinal background. Information was obtained from interviews and by reviewing studies of Chaoshan and Cantonese reported in the PubMed.

\section{Results}

\section{Ethnobotanical survey}

Through the investigation, we have found a total of 86 herbs, including 52 families and 82 genera (Table 1). Details of relative number of species per family as medicinal herbs in Chaoshan area are showed in Figure 1. Among Leguminosae accounted for 11, Compositae accounted for 5 and so on. The folk prescription aggregated into the editor, and the Chinese herbal medicine compilation and Chinese Pharmacopoeia contrast found Wikstroemia indica (Linn.) C. A. Mey, Ampelopsis brevipedunculata (Maxim.) Trautv., Bombax malabarica L. and other herbs to the methods used in the treatment of arthritis in both of the books were no record or record dosage and extraction methods are different. Of the 86 plants recorded, for most (37 species)

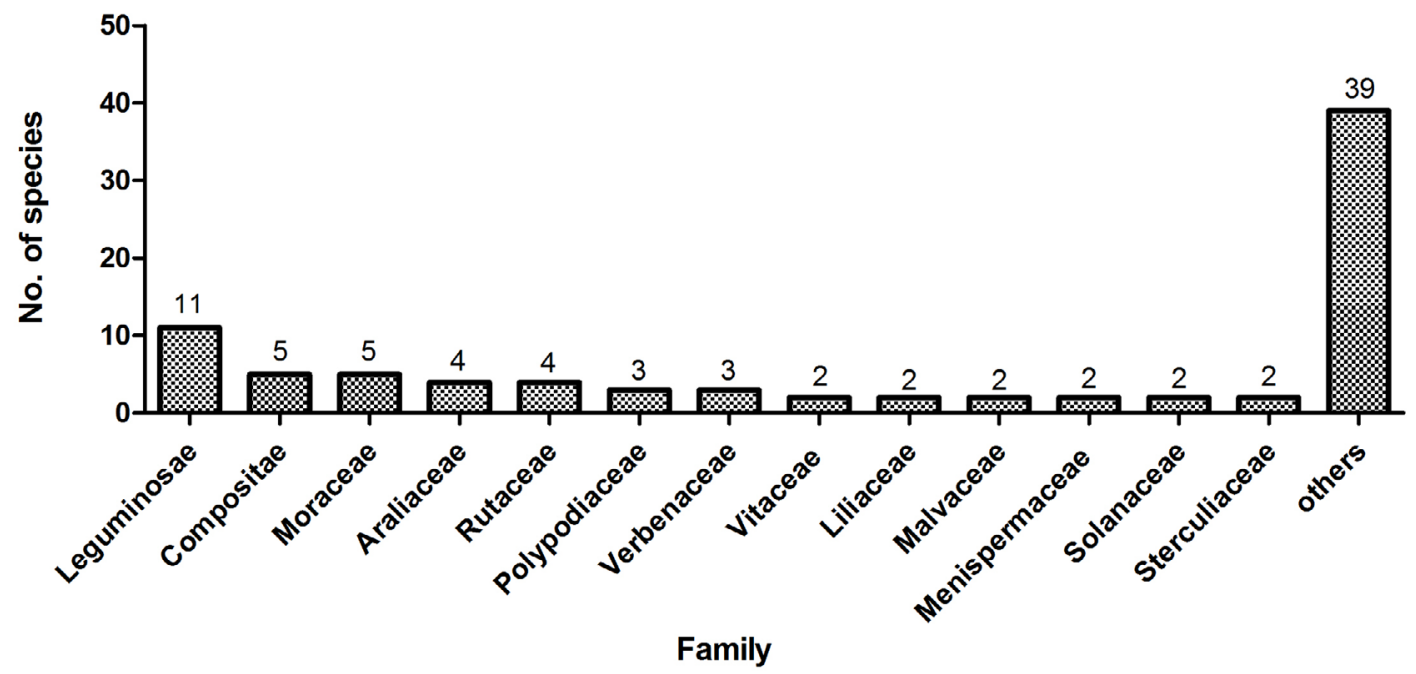

Figure 1: Relative number of species per family used as medicinal herbs by the ChaoShan area. 


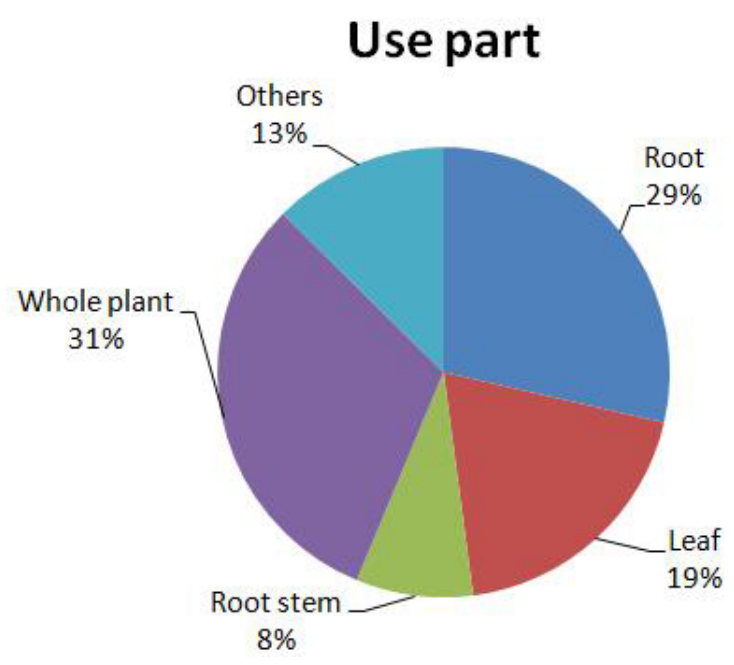

Figure 2: Form in which Chao Shan medicinal herbs are used.

the entire plant is used as medicine. Of the remaining species, for 34 only their roots are used, and for 23 only their leaves are used. Figure 2 displays the result of analysis of medicinal plant parts used to treat ailment.

\section{The pharmacological effects of herbal medicine reported in the PubMed}

Through in PubMed database search we investigate to the herbs in the treatment of arthritis of the research, we found 28 kinds of Chinese herbal medicine has done related pharmacological research. Among them, there are anti-inflammatory effects of 28 species, analgesia or ease the pain of a total of eight kinds of, and antibacterial, immune regulation and anti-hyperuricemia.

\section{Anti-inflammatory effect}

Narendhirakannan found that gynandra L. $150 \mathrm{mg} \mathrm{kg} /$ Cleome leaves methanol extract has significant anti-inflammatory activity in adjuvant arthritis rats [17-19] (Table 2). Yang caught sight of the ethanol and ethyl acetate extracts from Radix Toddaliae Asiaticae significantly reduced claws and joint swelling and spleen index is reduced, and reducing cytokines such as TNF-alpha, the concentration of IL-1, IL-6, and cytokines such as IL-10 was significantly higher than that of control group [61]. Matsui compounds of lansamide I, lansiumamide B, and SB-204900 from Clausena lansium (Lour.) Skeels (Rutaceae) markedly decreased histamine release. In addition, lansiumamide B- and SB204900-treated cells also reduced the protein and/or mRNA levels of TNF-a. The phosphorylation of p38 MAPK was markedly suppressed by SB-204900 [32]. Rodanant was found to be highly effective in the use of paniculata Murraya compounds (L.) Jack to stimulate monocyte inflammation on lipopolysaccharide [37]. The extract of Celastrus - (-) -Epiafzelechin has inhibitory effect on cyclooxygenase, and exhibited about 3-fold weaker inhibitory potency on the enzyme activity than indomethacin as a positive control. Furthermore, (-) -Epiafzelechin shows significant anti-inflammatory activity on carrageenin-induced mouse paw edema when the compound $(100 \mathrm{mg} / \mathrm{kg}$ ) was orally administrated at $1 \mathrm{~h}$ before carrageenin treatment [43]. Meng has adiscover on Saururus chinensis (Lour.) Baill., the subfraction 4 (SCF4) from the n-hexane layer of the ethanol extract of the aerial parts of S. chinensis significantly inhibited the production of nitrite and the expression of pro-inflammatory mediators via heme oxygenase-1 upregulation [52].

While Sauchinone, isolated from the roots of Saururus chinensis (LOUR.) BAILL., exhibited the vascular protective effects in human umbilical vein endothelial cells induced heme oxygenase (HO)1 expression [51]. And Kim, Cho's study showed that S. chinensis methanol extract has antioxidative and anti-inflammatory activities by enhancing antioxidative defense systems and suppressing NO production via the down-regulation of iNOS expression and NFkappaB activity $[49,50]$. Pereira reported Intraperitoneal injection of methanol extract of Bidens pilosa (PLN) can significantly reduce the size of the popliteal lymph node inflammation caused by zymosan [62]. Chih was found by contrast experiment that extracts $(500 \mathrm{mg} / \mathrm{kg})$ of $B$. pilosa $\mathrm{L}$. var. minor and $B$. pilosa $\mathrm{L}$. is more significantly decreased the paw edema induced by complete Freund's adjuvant than $B$. chilensis DC [63]. Lu's study results showed that a compound extracted from the rhizome of Smilax glabra, at non-cytotoxicity concentrations, significantly suppressed the production of nitric oxide (NO) and tumor necrosis factor- $\alpha$ (TNF- $\alpha$ ), as well as the mRNA expression of inducible nitric oxide synthase (iNOS) and TNF- $\alpha$ in LPS-induced RAW 264.7 cells, but did not affect interleukin-6 (IL-6) release or its mRNA expression. It seems related to its up-regulation of the phosphorylation of p65, extracellular signal-regulated kinases $1 / 2$ (ERK1/2) and c-Jun N-terminal kinase (JNK) [55]. Patel found the ethyl acetate (EtOAc) extract of Cassia occidentalis $\mathrm{L}$. (roots) $\left(\mathrm{IC}_{50}=21.3\right.$ to $43.1 \mathrm{micro} \mathrm{g} /$ $\mathrm{mL}$ ) and Mimosa pudica (whole plant) $\left(\mathrm{IC}_{50}=31.7\right.$ to 47.2 micro g/ $\mathrm{mL}$ ) and the dichloromethane (DCM) extract of Leucas cephalotes (whole plant) ( $\mathrm{IC}_{50}=46.8$ to $49.3 \mathrm{micro} \mathrm{g} / \mathrm{mL}$ ) exhibited significant anti-inflammatory activity by in vitro inhibition of the production of TNF-alpha, IL-1beta and NO in LPS stimulated RAW 264.7 cells. Furthermore, the five compounds isolated from the ethyl acetate extract of Cassia occidentalis roots were found to suppress LPS-induced IL1beta, TNF-alpha and NO production in a concentration-dependent manner in these cells at $\mathrm{IC}_{50}$ values ranging from 22.5 to 97.4 micro M. Emodin and chrysophanol were also found active in inhibiting pro-inflammatory cytokines in vivo [56]. Saleem found both aqueous and alcoholic extracts of the leaves Gendarussa vulgaris (G. vulgaris) Nees. showed significant anti-inflammatory property in vivo method that was estimated by human red blood cell membrane stabilisation (HRBC) method and in vivo method was estimated on the carrageenan induced paw oedema. And alcoholic extract at a concentration of 300 $\mathrm{mg} / \mathrm{mL}$ showed potent activity on comparing with the standard drug diclofenac sodium [57].

Chen's results showed that the ethanol extract of S. octophylla has significant dose-dependent anti-inflammatory and antinociceptive activities. And its five different polar fractions especially the $\mathrm{CHCl}_{3}$ fraction significantly inhibited the abdominal writhing induced by acetic acid and ear edema induced by xylene, also increased pain threshold in hot plate test in $120 \mathrm{~min}$ and reduced ticking times in formalin test. The ethanol extract of S. octophylla and the $\mathrm{CHCl}_{3}$ fraction demonstrated an anti-RA effect in a dose-dependent manner. The levels of TNF- $\alpha$, IL-1 $\beta$ and IL- 6 in ethanol extract $(600 \mathrm{mg} / \mathrm{kg})$ and $\mathrm{CHCl}_{3}$ fraction $(300 \mathrm{mg} / \mathrm{kg})$ groups were significantly lower than those of the model group [58]. Yang had isolated Nine new with anolides from the leaves of Datura metel L. All isolates were evaluated for in vitro anti-inflammatory potential using LPS-stimulated RAW 264.7 murine macrophages. Among them, compounds daturafolisides A, daturafolisides B, baimantuoluoside B, 12-deoxywithastramonolide exhibited significant inhibition of nitrite production. Compounds daturafolisides $\mathrm{C}$, daturafolisides $\mathrm{D}$, daturafolisides $\mathrm{F}$, and daturataurin 
Citation: Chen P, Zheng F, Zhang Y, Gao F, Chen Y, et al. (2016) Ethnobotanical Study of Medicinal Plants on Arthritis Used by Chaoshan in Guangdong, China. Med Chem (Los Angeles) 6: 715-723. doi: 10.4172/2161-0444.1000420

\begin{tabular}{|c|c|c|c|c|c|c|}
\hline No. & Latin name & Family & Local name & Part use & From of administration & Medicinal use \\
\hline 1 & Alangium chinense(Lour.) Rehd & Alangiaceae & Ba jiao feng & Root, Leaf, Flower & Vinum, Soup & $\mathrm{RA}$, traumatic injury \\
\hline 2 & Murraya paniculata(L.) Jack & Rutaceae & Jiu li xiang & Root, Leaf & Vinum, Soup & $\begin{array}{c}\text { Traumatic injury, RA, localized } \\
\text { anesthesia }\end{array}$ \\
\hline 3 & $\begin{array}{l}\text { Wikstroemia indica (Linn.) C. A. } \\
\text { Mey }\end{array}$ & Thymelaeaceae & Liao ge wang & Root, Leaf & Soup, Compress & $\begin{array}{l}\text { RA, Traumatic injury, Injury } \\
\text { blooding }\end{array}$ \\
\hline 4 & Kyllinga brevifolia Rottb & Cyperaceae & San jia cao & Whole plant & Vinum, Decoction & RA, Traumatic injury \\
\hline 5 & Smilax glabra Roxb & Liliaceae & Tu fu ling & Root,Stem & Soup & RA \\
\hline 6 & $\begin{array}{c}\text { Ampelopsis } \\
\text { brevipedunculata(Maxim.)Trautv. }\end{array}$ & Vitaceae & $\begin{array}{l}\text { Da hao shan } \\
\text { pu tao }\end{array}$ & Root, Stem, Leaf & Decoction & RA, Traumatic injury \\
\hline 7 & Waltheria americama L. & Sterculiaceae & He ta cao & Root,Whole plant & Decoction & Relieve pain and inflammation \\
\hline 8 & Toddalia asiatica (L.) Lam. & Rutaceae & $\begin{array}{c}\text { Fei long zhang } \\
\text { xue }\end{array}$ & Root, Leaf & Vinum, Decoction & RA, Traumatic injury \\
\hline 9 & Ipomoea pescaprae (L.) Sweet. & Convolvulaceae & Ma an teng & Whole plant & Vinum, Decoction, Soup & Rheumatic pain, Knee joint pain \\
\hline 10 & Bombax malabarica L. & Bombacaceae & Mu mian & Root, Stem, Bark & Decoction, Vinum & Traumatic injury \\
\hline 11 & Ficus simplicissima Lour. & Moraceae & Wu zhi mao tao & Root, Stem & Decoction & RA, Traumatic injury \\
\hline 12 & Impatiens balsamina $\mathrm{L}$. & Balsaminaceae & Feng xian hua & Flower & Decoction drink by liquor & RA \\
\hline 13 & Gendarussa vulgaris Nees. & Acanthaceae & $\begin{array}{l}\text { Wu gu huang } \\
\text { teng }\end{array}$ & Whole plant & Decoction & $\begin{array}{l}\text { Fracture, Sprain and contusion, } \\
\text { RA }\end{array}$ \\
\hline 14 & Ficus pandurata Hance. & Moraceae & Niu nai shu & Root, Leaf & Decoction drink by liquor & Traumatic injury \\
\hline 15 & Adenosma glutinosum(L.) Druce & Scrophulariaceae & Mao she & Whole plant & Decoction, compress & RA, Traumatic injury \\
\hline 16 & Callicarpa loureiri Hook. etArn & Verbenaceae & $\begin{array}{l}\text { Chang ye zi zhu } \\
\text { cao }\end{array}$ & Root, Stem & Decoction & RA, Traumatic injury \\
\hline 17 & Saurures chinensis (Lour.) Baill & Saururaceae & Shui lao & Root, Stem & Decoction & Diuretic swelling \\
\hline 18 & Lysimachia fortunei Maxim. & Primulaceae & Shui pu yin & Whole plant & Decoction & RA, Traumatic injury \\
\hline 19 & Pholidota chinensis Lindl & Orchidaceae & Shi xian tao & Bulb & Soup & RA \\
\hline 20 & Humata tyermanni S.Moore. & Davalliaceae & Shi qiu yin & Rhizome & Decoction & RA, Urticaria \\
\hline 21 & Lycoris radiate(L Herit) Herb & Amaryllidaceae & Shi suan & Bulb & Compress & RA, \\
\hline 22 & Phymatopsis hastata (thunb.) Kitag & Polypodiaceae & Long she hao & Whole plant & Vinum & Osteomyelitis \\
\hline 23 & $\begin{array}{l}\text { Clerodendron japonicum (Thunb) } \\
\text { Sweet. }\end{array}$ & Verbenaceae & Long chuang hua & Root, Leaf & Hot compress & $\begin{array}{l}\text { RA, Lumbar muscle strain, } \\
\text { Traumatic injury }\end{array}$ \\
\hline 24 & Selaginella uncinata (Desv.) Spring & Selaginellaceae & Long ling cao & Whole plant & Vinum & RA, Traumatic injury \\
\hline 25 & Bidens pilosa L. & Compositae & Si fang ku gu jing & Whole plant & Soup & Antiinflammatory, Antidiarrheal \\
\hline 26 & Chloranthus henryi Hemsl & Chloranthaceae & Si kuai wa & Root,Whole plant & Decoction & RA, Traumatic injury \\
\hline 27 & Siegesbeckia glabrescens Makino. & Compositae & Mu jing cao & Whole plant & Decoction & RA, Limbs anesthesia \\
\hline 28 & Cleome gynandra L. & Capparidaceae & Bai hua chou cao & Seed or Whole plant & Compress & RA \\
\hline 29 & Acanthopanax trifoliatus (L.)Merr & Araliaceae & Bai le & Root, Leaf & Decoction & RA \\
\hline 30 & $\begin{array}{l}\text { Pterospermum heterophyllum } \\
\text { Hance. }\end{array}$ & Sterculiaceae & Ban feng he & Root stem & Decoction, Vinum & $\begin{array}{l}\text { RA } \\
\text { Lumbar muscle strain traumatic } \\
\text { injury, Sprain and contusion }\end{array}$ \\
\hline 31 & Eupatorium chinense L. & Compositae & Duo xu gong & Root, Leaf & Decoction, add salt & RA, Bronchitis \\
\hline 32 & Millettia dielisana Harms ex Diels. & Leguminosae & Xue feng gen & Root rattan & Decoction and vinum & $\begin{array}{l}\text { RA, Traumatic injury, } \\
\text { Limbs anesthesia }\end{array}$ \\
\hline 33 & Inula cappa DC. & Compositae & Chong tian bai & Root,Whole plant & Soup & $\begin{array}{c}\text { Rheumatism edema, Traumatic } \\
\text { injury }\end{array}$ \\
\hline 34 & Artocarpus hypargyraea Hance & Moraceae & Hong she geng & Root & Decoction or soak in Liquor & RA \\
\hline 35 & Ardisiae gigantifoliae Stapf. & Myrsinaceae & Zou ma tai & $\begin{array}{l}\text { Root,Leaf,Whole } \\
\text { plant }\end{array}$ & Decoction & RA, Traumatic injury \\
\hline 36 & $\begin{array}{c}\text { Cunninghamia lanceolata (Lamb.) } \\
\text { Hook }\end{array}$ & Taxodiaceae & Sang & $\begin{array}{l}\text { Root, Bark, Fruit, } \\
\text { Wood }\end{array}$ & Decoction & RA, Traumatic injury, \\
\hline 37 & Xanthium Sibiricum Patr. Ex Widd. & Compositae & Can ger zi & Fruit, Whole plant & Decoction & RA \\
\hline 38 & $\begin{array}{l}\text { Moghania philippinensis (Merr. et } \\
\text { Rolfe) Li }\end{array}$ & Leguminosae & Ding di gen & Root, Leaf & Decoction & $\begin{array}{l}\text { Lumbar muscle strain, Traumatic } \\
\text { injury }\end{array}$ \\
\hline 39 & $\begin{array}{c}\text { Ganoderma lucidum(Leyss. ex Fr.) } \\
\text { Karst. }\end{array}$ & Polyporaceae & Ling zhi & Sporocarp & Tincture & RA, Bronchitis \\
\hline 40 & Rhynchosioa volubilis Lour. & Leguminosae & $\begin{array}{l}\text { Ji zai mu } \\
\text { zhougen }\end{array}$ & Whole plant & Soup & $\mathrm{RA}$, Llumbar muscle strain \\
\hline 41 & Paederia scandens (Lour.) Merr. & Rubiaceae & Ji shi teng & Whole plant & Vinum & RA \\
\hline
\end{tabular}


Citation: Chen P, Zheng F, Zhang Y, Gao F, Chen Y, et al. (2016) Ethnobotanical Study of Medicinal Plants on Arthritis Used by Chaoshan in Guangdong, China. Med Chem (Los Angeles) 6: 715-723. doi: 10.4172/2161-0444.1000420

\begin{tabular}{|c|c|c|c|c|c|c|}
\hline 42 & Abrus cantoniensis Hance. & Leguminosae & Ji gu cao & $\begin{array}{c}\text { Whole plant(execpt } \\
\text { seed) }\end{array}$ & Decoction & RA \\
\hline 43 & Achyranthes aspera L. & Amarantaceae & Ji cuo bi & Root or Whole plant & $\begin{array}{l}\text { Decoction and drink by } \\
\text { Liquor }\end{array}$ & RA \\
\hline 44 & Liquidambar formosana Hance & Hamamelidaceae & Feng shu & $\begin{array}{l}\text { Root, Leaf, Fruit- } \\
\text { resin }\end{array}$ & Decoction & RA \\
\hline 45 & Aralia chinensis $\mathrm{L}$. & Araliaceae & $\mathrm{Ci}$ jiang mu & Root,Stem & Soup & RA, Traumatic injury \\
\hline 46 & Zanthoxylum avicennae (Lam.)DC & Rutaceae & Ci cang gen & Root,Leaf & Decoction, soak in liquor & RA, Traumatic injury \\
\hline 47 & Desmodium caudatum (Thunb.)DC. & Leguminosae & Mo cao & Root or Whole plant & Vinum & RA \\
\hline 48 & Glechoma longituba(Nakai) Kupr. & Labiatae & $\begin{array}{c}\text { Ruan zhi jie gu } \\
\text { xiao }\end{array}$ & Whole plant & Drink by liquor & RA, Fracture \\
\hline 49 & Rubus parvifolius L. & Rosaceae & Hu mu gen & Root & Alcohol extraction & $\begin{array}{l}\text { Antiinflammatory, Analgesic, } \\
\text { Insecticide }\end{array}$ \\
\hline 50 & $\begin{array}{c}\text { Podocarpus macrophyllus (Thunb.) } \\
\text { D. Don }\end{array}$ & Podocarpaceae & Luo han song & Root,Leaf & Fire with liquor Compress & RA, Traumatic injury, Fracture \\
\hline 51 & Salix babylonica L. & Salicaceae & Cui liu & $\begin{array}{l}\text { Branch Root Bark, } \\
\text { Fibril }\end{array}$ & Decoction & RA \\
\hline 52 & Uraria crinite Desv. & Leguminosae & Hu li wei & Whole plant & Fumigant & Bronchitis, RA, Traumatic injury \\
\hline 53 & Urena procumbens $\mathrm{L}$. & Malvaceae & Gou jiao ji & Whole plant & Soup & RA, Traumatic injury \\
\hline 54 & Solanum indicum $\mathrm{L}$. & Solanaceae & Ci qie & Root or Whole plant & Soup & RA, Traumatic injury \\
\hline 55 & Celastrus orbiculatus Thunb. & Celastraceae & Nan she teng & Root, Rattan & Vinum & RA, Traumatic injury \\
\hline 56 & Oxalis corniculata L. & Oxalidaceae & Xian suan cao & Whole plant & Vinum & Traumatic injury, RA \\
\hline 57 & Drynaria fortune (kunze)J.Smith & Polypodiaceae & Gu sui bu & Rhizome & Vinum, Decoction & Traumatic injury, Fracture, RA \\
\hline 58 & Cymbopogon citrates (DC.)Stapf. & Gramineae & Xiang mao & Whole plant & Compress & Pain from rheumatism \\
\hline 59 & Datura metel L. & Solanaceae & Yang jin hua & Flower, Leaf, Seed & Vinum, Soup & Chronic bronchitis, RA \\
\hline 60 & Schefflera octophylla (Lour.) Harms & Araliaceae & Ya jiao mu & Bark,Leaf,Root & Vinum & RA, Sciatica, Traumatic injury \\
\hline 61 & Millettia speciosa champ. & Leguminosae & $\begin{array}{l}\text { Dao diao jin } \\
\text { zhong }\end{array}$ & Root & Decoction & $\begin{array}{c}\text { RA, Lumbar muscle strain, Chronic } \\
\text { bronchitis, } \\
\text { Traumatic injury }\end{array}$ \\
\hline 62 & Clerodendrum fragrans Vent. & Verbenaceae & Chou mo li & Leaf, Root & Soup, Decoction & RA, Sprain and Contusion \\
\hline 63 & $\begin{array}{l}\text { Cissus modecoides Planch var. } \\
\text { subintegra Gagnep. }\end{array}$ & Vitaceae & Feng teng tou & Root & Decoction, Compress & RA, Lumbar muscle strain \\
\hline 64 & Morus alba L. & Moraceae & Sang bai pi & Branch & Decoction & Rheumatism edema, Joint pain \\
\hline 65 & Clausena lansium (Lour.) Skeels. & Rutaceae & Huang shu pi & Root & Decoction & Root treat rheumatic arthritis, \\
\hline 66 & Gymnema sylvestre(Retz.) schult. & Asclepiadaceae & Gua xue zai teng & Stem, Leaf, Root & Compress & RA, Traumatic injury \\
\hline 67 & Desmodium pulchellum(L.) Benth & Leguminosae & Pai qian cao & Whole plant & Soup & RA \\
\hline 68 & $\begin{array}{l}\text { Hedera nepalensis K,Koch var.. } \\
\text { sinensis (Tobl.) Rehd }\end{array}$ & Araliaceae & Chang chun teng & stem, Whole plant & Fumigant and washing & $\begin{array}{l}\text { RA, Sciatica, Osteomyelitis, } \\
\text { Traumatic injury }\end{array}$ \\
\hline 69 & $\begin{array}{c}\text { Pratia nummularia A. Brown. et } \\
\text { Aschers. }\end{array}$ & Campanulaceae & $\begin{array}{l}\text { Tong cui yu dai } \\
\text { cao }\end{array}$ & Whole plant & Decoction & RA, Traumatic injury \\
\hline 70 & Ranunculus sceleratus L. & Ranunculaceae & Jia qin cai & Whole plant & Decoction & RA \\
\hline 71 & Desmos chinensis Lour. & Annonaceae & Jia yin zhua & Root, Whole plant & Decoction & RA, Traumatic injury \\
\hline 72 & Stephania longa Lour. & Menispermaceae & Li bi teng & Whole plant & Decoction & RA, Sciatica \\
\hline 73 & Cassia occidentalis L. & Leguminosae & Wang jiang nan & Whole plant & Decoction & RA, Traumatic injury \\
\hline 74 & Lycopodium cernuum L. & Lycopodiaceae & Lu jiao mao & Whole plant & Decoction & RA, Traumatic injury \\
\hline 75 & Desmodium triquetrum(L.)DC. & Leguminosae & Hu lu cha & Whole plant & Decoction & Waist pain \\
\hline 76 & Melastoma candidum D.Don. & Melastomataceae & Yin zhi pu bi & Root, Leaf & Decoction & Migraine, RA, Traumatic injury \\
\hline 77 & Caesalpinia minax Hance & Leguminosae & La ba ci & Root, Stem, Leaf & Decoction & RA, Traumatic injury fracture \\
\hline 78 & $\begin{array}{l}\text { Cocculus sarmenntosus (Lour.) } \\
\text { Diels }\end{array}$ & Menispermaceae & Yi dou jin & Root & Decoction & RA, Sciatica \\
\hline 79 & Pistia stratiotes $\mathrm{L}$. & Araceae & Fan pin & Leaf & Decoction & Rheumatism Edema, RA \\
\hline 80 & Mirabilis jalapa L. & Nyctaginaceae & Gu shou hua gen & Root, Leaf & Decoction & Acute arthritis \\
\hline 81 & Basella rubra $\mathrm{L}$. & Basellaceae & Pu ten gcai & Whole plant & Soup & RA, Fracture, Traumatic injury \\
\hline 82 & Berchemia lineata DC. & Rhamnaceae & Shu ru gen & Root & Soup & Traumatic injury, RA \\
\hline 83 & Smilax china L. & Liliaceae & Hao ke ci & Rhizome & Vinum, Decoction & RA, Traumatic injury, Cancer \\
\hline 84 & Ficus microcarpa L.f. & Moraceae & Rong shu & Root, Aerial root & Soup, Sirup & RA, Bronchitis \\
\hline 85 & $\begin{array}{c}\text { Malvastrum coromandelianum (L.) } \\
\text { Garcke }\end{array}$ & Malvaceae & Sai kui & Whole plant & Soup & RA, Traumatic injury \\
\hline 86 & $\begin{array}{c}\text { Cinnamomum parthenoxylon (Jack) } \\
\text { Nees. }\end{array}$ & Lauraceae & Zhang shu & $\begin{array}{l}\text { Root, Stem, Leaf, } \\
\text { Bark }\end{array}$ & Decoction & Traumatic injury, RA \\
\hline
\end{tabular}

Note: RA: Rheumatic Arthritis, Traumatic injury

Table 1: Medicinal plants used by Chaoshan. 
Citation: Chen P, Zheng F, Zhang Y, Gao F, Chen Y, et al. (2016) Ethnobotanical Study of Medicinal Plants on Arthritis Used by Chaoshan in Guangdong, China. Med Chem (Los Angeles) 6: 715-723. doi: 10.4172/2161-0444.1000420

\begin{tabular}{|c|c|c|}
\hline Latin & Pharmacological Action & Reference \\
\hline Cleome gynandra L. & Anti-inflammatory & [17-19] \\
\hline Pterospermum heterophyllum Hance. & Anti-inflammatory & [20] \\
\hline Eupatorium chinense L. & Anti-inflammatory & [21] \\
\hline Toddalia asiatica (L.) Lam. & Anti-inflammatory, Analgesia, Antibacterial & {$[22,23]$} \\
\hline Impatiens balsamina L. & Antibacterial, Analgesia & [24] \\
\hline Mirabilis jalapa L. & Antibacterial & {$[25,26]$} \\
\hline Waltheria americama L. & Analgesia & [27] \\
\hline Smilax china L. & Anti-inflammatory, Uricotelic, Analgesia & [28-30] \\
\hline Clausena lansium (Lour.) Skeels. & Anti-inflammatory, Analgesia & [31-33] \\
\hline Achyranthes aspera L. & Immunomodulatory effects & [34] \\
\hline Abrus cantoniensis Hance. & Immunomodulatory effects & [35] \\
\hline Ranunculus sceleratus L. & Anti-inflammatory & {$[36]$} \\
\hline Murraya paniculata(L.) Jack & Antibacterial, Anti-inflammatory & [37] \\
\hline Caesalpinia minax Hance & Anti-inflammatory & {$[38,39]$} \\
\hline Ganoderma lucidum (Leyss. ex Fr.) Karst. & Immunomodulatory effects & [40] \\
\hline Ipomoea pescaprae (L.) Sweet. & Analgesic & [41] \\
\hline Desmodium caudatum (Thunb.)DC. & Anti-inflammatory, Analgesia & [42] \\
\hline Celastrus orbiculatus Thunb. & Anti-inflammatory & [43] \\
\hline Ficus pandurata Hance. & Analgesia & [44] \\
\hline Basella rubra L. & Immunomodulatory effects & [45] \\
\hline Morus alba L. & Antibacterial, Immunomodulatory effects, Uricotelic & [46-48] \\
\hline Saururus chinensis (Lour.) Baill & Anti-inflammatory & [49-52] \\
\hline Bidens pilosa L. & Anti-inflammatory, Antibacterial & {$[53,54]$} \\
\hline Smilax glabra Roxb & Anti-inflammatory & [55] \\
\hline Cassia occidentalis L. & Anti-inflammatory & {$[56]$} \\
\hline Gendarussa vulgaris Nees. & Anti-inflammatory & [57] \\
\hline Schefflera octophylla (Lour.) Harms & Analgesia, Anti-inflammatory & [58] \\
\hline Datura metel L. & Anti-inflammatory, Antifungal & {$[59,60]$} \\
\hline
\end{tabular}

Table 2: The pharmacological effects of herbal medicine.

B presented moderate inhibitory activities with values of $\mathrm{IC}_{50}$ at 59.0 , $52.8,71.2$, and $53.1 \mu \mathrm{M}$, while the rest compounds displayed weak suppressive effect. In addition, they found Compounds dmetelins A, dmetelins D, 7a,27-dihydroxy-1-oxo-witha-2,5,24-trienolide isolated from the leaves of Datura metel L. (Solanaceae) also showed significant inhibitory activities on lipopolysaccharide (LPS)-induced nitric oxide (NO) production in RAW264.7 cells, and compounds dmetelins B and dmetelins $\mathrm{C}$ showed moderate inhibitory activities with $\mathrm{IC}_{50}$ values of $17.8,11.6,14.9,33.3$ and $28.6 \mu \mathrm{M}$, respectively.

\section{Analgesia}

Kariuki HN's experiment in 2013 showed, in the early phase of the formalin test, the $100 \mathrm{mg} / \mathrm{kg}$ dose showed no significant antinociceptive activity while the $200 \mathrm{mg} / \mathrm{kg}$ showed significant $(\mathrm{p}<0.01)$ antinociceptive activity. The $100 \mathrm{mg} / \mathrm{kg}$ dose showed highly significant antinociceptive activity $(\mathrm{p}<0.001)$ in the late phase of the formalin test while the $200 \mathrm{mg} / \mathrm{kg}$ dose showed no significant antinociceptive activity. A reduction in carragenin induced acute inflammation paw oedema was significant $(\mathrm{p}<0.01)$ following administration of 100 $\mathrm{mg} / \mathrm{kg}$ dose but not with the $200 \mathrm{mg} / \mathrm{kg}$ dose. The study therefore lends support to the anecdotal evidence for use of T. asiatica in the management of painful and inflammatory conditions [23]. Oku H's results showed 1,4-naphthoquinone sodium salts, sodium 3-hydroxide2[[sodium3-hydroxide-1,4-dioxo(2-naphthyl)] ethyl]naphthalene-1,4dione (impatienolate) and sodium 2-hydroxide-3-(2-hydroxyethyl) naphthalene-1,4-dione (balsaminolate) isolated from the corolla of Impatiens balsamina L exhibited Significant selective cyclooxygenase-2 (COX-2) inhibitory activities [24]. Muthuraman A 's study showed that rats administered the hydroalcoholic extract of Acorus calamus could increase the levels of superoxide anion, total calcium and myeloperoxidase activity significantly in chronic constriction injury of sciatic nerve induced thermal, radiant, mechanical hyperalgesia and thermal, chemical, tactile allodynia. Moreover, HAE-AC attenuated chronic constriction injury induced by development of painful behavioural, biochemical and histological changes in a dose dependent manner similar to that of pregabalin serving as positive control [64]. Khunakornvichaya A found oral administration of M. alba stem extract ( 56 and $560 \mathrm{mg} / \mathrm{kg}$ ) significantly attenuated joint pain as indicated by a significant $(\mathrm{p}<0.05)$ increase in the values of percent weight borne on the operated hind limb for the OA-induced groups that received M. alba stem extract at 56 and $560 \mathrm{mg} / \mathrm{kg}$ when compared to those of the vehicle-treated OA-induced group. Moreover, a significant improvement in the Mankin score was also observed in rats treated with $560 \mathrm{mg} / \mathrm{kg} \mathrm{M}$. alba stem extract, which was in agreement with its pain-relieving effect. The results showed that $M$. alba stem extract exhibited an anti-nociceptive effect as well as cartilage protection in the ACLT-induced rat model of OA47. Ma KJ found oral administration of Desmodium caudatum (Thunb.) DC significantly and dose-dependently inhibited the writhing responses in mice, increased reaction time in mice in the hot-plate test. Furthermore, no death was observed when mice were orally administered DCE up to $40 \mathrm{~g} / \mathrm{kg}$ [42]. Khedr AI did a molecule docking with compounds 3b-acetoxy-11amethoxy-olean12-ene, ficupanduratin A, stigmastane-3,6-dione isolated from Ficus pandurata that exhibited good affinity towards the CB2 receptor, with displacement values of $69.7,62.5$ and $86.5 \%$, respectively [44]. Chen's results showed that the ethanol extract of Schefflera octophylla has significant dose-dependent antinociceptive activities. And its five different polar fractions especially the $\mathrm{CHCl}_{3}$ fraction significantly inhibited the abdominal writhing induced by acetic acid and ear edema induced by xylene, also increased pain threshold in hot plate test in 120 
min and reduced ticking times in formalin test [58]. The antinociceptive effects of the methanolic extract and two fractions obtained from aerial parts of Ipomoea pescaprae (L.) Sweet. exhibited considerable antinociceptive activity against two classical models of pain in mice. Methanolic extract presented a calculated $\mathrm{ID}_{50}$ value of $33.8 \mathrm{mg} / \mathrm{kg}$, i.p. against writhing test and also inhibited both phases of pain (neurogenic and inflammatory) of the formalin test with $\mathrm{ID}_{50}$ of 37.7 and $12.5 \mathrm{mg} /$ $\mathrm{kg}$, i.p. for the first and second phase, respectively [41].

\section{Uricotelic}

Chen studies showed that ethyl acetate fraction from Smilax china L. showed a significant anti-hyperuricemic activity in hyperuricemic mice compared with petroleum ether, chloroform, n-butanol and residual ethanol fraction fractions. Caffeic acid, resveratrol, rutin and oxyresveratrol isolated from EAF showed different inhibitory activities on xanthine oxidase in vitro, with the $\mathrm{IC}_{50}$ values of $42.60,37.53$, 42.20 and $40.69 \mu \mathrm{M}$, respectively, and exhibited competitive or mixed inhibitory actions [28]. Different dosages of astilbin which isolated from the rhizome of Smilax china L. $(1.25,2.5$, and $5.0 \mathrm{mg} / \mathrm{kg})$ were administered to $10 \%$ fructose-induced hyperuricemic rats. In Chen studies, the results demonstrated that astilbin significantly decreased the serum uric acid (Sur) level by increasing the urinary uric acid (Uur) level and fractional excretion of urate (FEUA) but not inhibiting the xanthine oxidase (XOD) activity [65]. The effective substance of Morus alba L. could promote the excretion of uric acid through different mechanisms in rats and mice, reduce the concentration of uric acid in the body [66,67].

\section{Immunomodulatory effects}

Narayan found that PCA feed urethane primed lung tissues showed down regulated expression of pro-inflammatory cytokines IL-1 $\beta$, IL-6 and TNF- $\alpha$ along with TFs, NF- $\mathrm{BB}$ and Stat 3 while the expression of pro-apoptotic proteins Bax and p53 were enhanced in PCA feed urethane primed lung tissues [34]. In Rubel R's test G. lucidum was able to increase interferon-gamma (IFN-gamma) concentration but reduced $\mathrm{CD} 3(+)$ and $\mathrm{CD} 8(+)$ spleen lymphocytes. in vivo. Ex-vivo, IFN-gamma; and interleukin-10 levels were increased and the tumor necrosis factor-alpha (TNF-alpha) level was reduced by peritoneal macrophages from mice fed with G. lucidum [40]. An extract from Basella rubra (B. rubra) L polysaccharide in -BRP-4, can generate $\mathrm{NO}$ at a concentration of 10 to $100 \mathrm{~g} / \mathrm{mL}$. The phagocytic activity of macrophage was enhanced in BRP-4 treated RAW264.7 cells. BRP-4 combined with concanavalin A (Con A) provided obvious promotion and strengthening of the proliferation of the splenocytes [45]. M. alba L. fruit extract could stimulated the production of cytokines, nitric oxide (NO) and tumour necrosis factor- $\alpha$ (TNF- $\alpha$ ) and tumoricidal properties of macrophages. MFE activated macrophages through the mitogen-activated protein kinase (MAP Kinase) and nuclear factor$\kappa B(N F-\kappa B)$ signaling pathways downstream from toll-like receptor (TLR) 4. MFE was shown to exhibit cytotoxicity of CT26 cells via the activated macrophages, even though MFE did not directly affect CT26 cells. In a xenograft mouse model, MFE significantly enhanced anticancer activity combined with 5-fluorouracil and markedly promoted splenocyte proliferation, natural killer (NK) cell activity, cytotoxic T lymphocyte (CTL) activity and IFN- $\gamma$ production. Immunoglobulin $\mathrm{G}$ (IgG) antibody levels were significantly increased [46].

\section{Discussion and Conclusion}

The present investigation revealed that Chaoshan in Guangdong are using 86 plant species belonging to 82 genera in 52 families for arthritis. Most species are collected locally. In previous Chaoshan Chinese herbal medicine culture inheritance is survive on "father son" mode of inheritance. However, with the advance of the process of urbanization in the Chaoshan area, many of the older generation Chaoshan herbalist facing no successor predicament, because a new generation of Chaoshan young people unwilling to engage in grass pharmacists job. Also, the fast disappearance of traditional culture and natural resources due to urbanization and industrialization suggests that unrecorded information may be lost forever. Thus, We urgently need document systematically the medical knowledge of Chaoshan.

In relation to plant use, 72 species of the plants recorded in this study were found to be used for treating rheumatic arthritis, part of them used for analgesia, anti-inflammatory. Through the analysis of the results of a search of PubMed database we obtain, reported in the literature of Chinese herbal medicine, more than half have antiinflammatory effect and analgesic effect of the herbs in the quantity ranks the second. And the clinical treatment of arthritis the method is by non-steroidal anti-inflammatory drugs to relieve inflammation and pain of patients.

In search of the 86 Chaoshan herbs, there are 28 herbs pharmacological studies on treatment of arthritis, indicating that there are still many Chaoshan herbal undetected and research. Chaoshan area is located along the coast, because of climate and diet, the natives are more likely to suffer from arthritis, rheumatoid arthritis. In the struggle to perennial and arthritis, this kind of disease, the Chaoshan area of the ancestors left a number of therapeutic arthritis remedies and herbal knowledge. Through to these knowledges were classified and unified will of Chaoshan area of herbal medicine culture inheritance and development of therapeutic arthritis drugs have very good help.

\section{Acknowledgments}

This work was supported by the grants from the Natural Science Foundation of Guangdong Province, China (No.2015A030313432), the Science and Technology Project of Guangdong Province, China (No.2016B090918129), and the Department of education, Guangdong government under the top-tier university development scheme for research and control of infectious diseases.

\section{References}

1. Colmegna I, Cuchacovich R, Espinoza LR (2004) HLA-B27-associated reactive arthritis: pathogenetic and clinical considerations. Clin Microbiol Rev 17: 348369.

2. Hamdulay SS, Glynne SJ, Keat A (2006) When is arthritis reactive? Postgrad Med J 82: 446-453.

3. Palazzi C, Olivieri I, D'Amico E, Pennese E, Petricca A (2004) Management of reactive arthritis. Expert Opin Pharmacother 5: 61-70.

4. Reveille JD, Arnett FC (2005) Spondyloarthritis: update on pathogenesis and management. Am J Med 118: 592-603.

5. Ballara S, Taylor PC, Reusch P, Marmé D, Feldmann M, et al. (2001) Raised serum vascular endothelial growth factor levels are associated with destructive change in inflammatory arthritis. Arthritis \& Rheumatism 44: 2055-2064.

6. Brennan FM, Browne KA, Green PA, Jaspar JM, Maini RN, et al. (1997) Reduction of serum matrix metalloproteinase 1 and matrix metalloproteinase 3 in rheumatoid arthritis patients following anti-tumour necrosis factor-alpha (cA2) therapy. Rheumatology 36: 643-650.

7. Charles P, Elliott MJ, Davis D, Potter A, Kalden JR, et al. (1999) Regulation of cytokines, cytokine inhibitors, and acute-phase proteins following anti-TNF- $\alpha$ therapy in rheumatoid arthritis. J Immunol 163: 1521-1528.

8. Feldmann M, Brennan FM, Maini RN (1996) Role of cytokines in rheumatoid arthritis. Ann Rev Immunol 14: 397-440

9. Paleolog EM, Young S, Stark AC, McCloskey RV, Feldmann M, et al. (1998) Modulation of angiogenic vascular endothelial growth factor by tumor necrosis factor $\alpha$ and interleukin1 in rheumatoid arthritis. Arthritis \& Rheumatism 41: 
Citation: Chen P, Zheng F, Zhang Y, Gao F, Chen Y, et al. (2016) Ethnobotanical Study of Medicinal Plants on Arthritis Used by Chaoshan in Guangdong, China. Med Chem (Los Angeles) 6: 715-723. doi: 10.4172/2161-0444.1000420

1258-1265.

10. Lee JC, Laydon JT, McDonnell PC, Gallagher TF, Kumar S, et al. (1994) A protein kinase involved in the regulation of inflammatory cytokine biosynthesis. Nature 372: 739-746.

11. Goldfeld AE, Strominger JL, Doyle C (1991) Human tumor necrosis factor alpha gene regulation in phorbol ester stimulated $T$ and $B$ cell lines. J Exp Med 174: 73-81.

12. McCaffrey PG, Goldfeld AE, Rao A (1994) The role of NFATp in cyclosporin A-sensitive tumor necrosis factor-alpha gene transcription. J Biol Chem 269: 30445-30450.

13. Shakhov AN, Collart MA, Vassalli P, Nedospasov SA, Jongeneel CV (1990) Kappa B-type enhancers are involved in lipopolysaccharide-mediated transcriptional activation of the tumor necrosis factor alpha gene in primary macrophages. J Exp Med 171: 35-47.

14. Bondeson J, Browne KA, Brennan FM, Foxwell BM, Feldmann M (1999) Selective regulation of cytokine induction by adenoviral gene transfer of $\mathrm{IKBa}$ into human macrophages: lipopolysaccharide-induced, but not zymosaninduced, proinflammatory cytokines are inhibited, but IL-10 is nuclear factor-KB independent. J Immunol 162: 2939-2945.

15. Brennan FM, Hayes AL, Ciesielski CJ, Green P, Foxwell BM, et al. (2002) Evidence that rheumatoid arthritis synovial $T$ cells are similar to cytokine activated T cells: Involvement of phosphatidylinositol $3 \square$ kinase and nuclear factor KB pathways in tumor necrosis factor a production in rheumatoid arthritis. Arthritis \& Rheumatism 46: 31-41.

16. Wu X (1998) Resources of medicinal plants and their conservation and utilization in Shantou area of Guangdong. Journal of Hanshan Normal University, pp: 124-134

17. Narendhirakannan RT, Kandaswamy M, Subramanian S (2005) Antiinflammatory activity of Cleome gynandra $\mathrm{L}$. on hematological and cellular constituents in adjuvant-induced arthritic rats. J Med Food 8: 93-99.

18. Narendhirakannan RT, Subramanian S, Kandaswamy M (2005) Free radical scavenging activity of Cleome gynandra L. leaves on adjuvant induced arthritis in rats. Mol Cell Biochem 276: 71-80.

19. Narendhirakannan RT, Subramanian S, Kandaswamy M (2007) Antiinflammatory and lysosomal stability actions of Cleome gynandra L. studied in adjuvant induced arthritic rats. Food Chem Toxicol 45: 1001-1012.

20. Li S, Shi Y, Shang XY, Cui BS, Yuan Y, et al. (2009) Triterpenoids from the roots of Pterospermum heterophyllum Hance. J Asian Nat Prod Res 11: 652657.

21. Itoh T, Ohguchi K, Nozawa Y, Akao Y (2009) Intracellular glutathione regulates sesquiterpene lactone-induced conversion of autophagy to apoptosis in human leukemia HL60 cells. Anticancer Res 29: 1449-1457.

22. Watanabe A, Kumagai M, Mishima T, Ito J, Otoki Y, et al. (2015) Toddaculin, Isolated from of Toddalia asiatica (L.) Lam., Inhibited Osteoclastogenesis in RAW 264 Cells and Enhanced Osteoblastogenesis in MC3T3-E1 Cells. PloS one 10: e0127158.

23. Kariuki HN, Kanui TI, Yenesew A, Patel N, Mbugua PM (2013) Antinocieptive and anti-inflammatory effects of Toddalia asiatica (L) Lam.(Rutaceae) root extract in Swiss albino mice. Pan Afr Med J 14: 133-138.

24. Oku H, Ishiguro K (2002) Cyclooxygenase-2 inhibitory 1, 4-naphthoquinones from Impatiens balsamina L. Biol Pharm Bull 25: 658-660.

25. Cammue BP, De Bolle MF, Terras FR, Proost P, Van Damme J, et al. (1992) Isolation and characterization of a novel class of plant antimicrobial peptides form Mirabilis jalapa L. seeds. J Biol Chem 267: 2228-2233.

26. De Bolle MF, Eggermont K, Duncan RE, Osborn RW, Terras FR, et al. (1995) Cloning and characterization of two CDNA clones encoding seed-specific antimicrobial peptides from Mirabilis jalapa L. Plant Mol Biol 28: 713-721.

27. Zongo F, Ribuot C, Boumendjel A, Guissou I (2013) Botany, traditional uses, phytochemistry and pharmacology of Waltheria indica L.(syn. Waltheria americana): a review. J Ethnopharmacol 148: 14-26.

28. Chen L, Yin H, Lan Z, Ma S, Zhang C, et al. (2011) Anti-hyperuricemic and nephroprotective effects of Smilax china L. J Ethnopharmacol 135: 399-405.

29. Lü Y, Chen D, Deng J, Tian L (2003) Effect of Smilax china on adjunctive arthritis mouse. Zhong Yao Cai 26: 344-346.

30. Yu HJ, Shin J, Lee SO, Kwon KH, Cho SD (2014) Extracellular signal-regulated kinase inhibition is required for methanol extract of Smilax china L.-induced apoptosis through death receptor 5 in human oral mucoepidermoid carcinoma cells. Mol Med Reports 9: 663-668.

31. Adebajo AC, Iwalewa EO, Obuotor EM, Ibikunle GF, Omisore NO et al. (2009) Pharmacological properties of the extract and some isolated compounds of Clausena lansium stem bark: anti-trichomonal, antidiabetic, anti-inflammatory, hepatoprotective and antioxidant effects. J Ethnopharmacol 122: 10-19.

32. Matsui T, Ito C, Furukawa H, Okada T, Itoigawa M (2013) Lansiumamide $B$ and SB-204900 isolated from Clausena lansium inhibit histamine and TNF- $\alpha$ release from RBL-2H3 cells. Inflammation Research 62: 333-341.

33. Shen DY, Chan YY, Hwang TL, Juang SH, Huang SC, et al. (2014) Constituents of the Roots of Clausena lansium and Their Potential Anti-inflammatory Activity. J Nat Prod 77: 1215-1223.

34. Narayan C, Kumar A (2014) Antineoplastic and immunomodulatory effect of polyphenolic components of Achyranthes aspera (PCA) extract on urethane induced lung cancer in vivo. Mol Biol Rep 41: 179-191.

35. Wu S, Fu X, Brennan MA, Brennan CS, Chun C (2016) The Effects of Different Purifying Methods on the Chemical Properties, in Vitro Anti-Tumor and Immunomodulatory Activities of Abrus cantoniensis Polysaccharide Fractions. Int J Mol Sci 17: 511-526.

36. Prieto JM, Recio MC, Giner RM, Máñez S, Ríos JL (2003) Pharmacological approach to the pro-and anti-inflammatory effects of Ranunculus sceleratus $\mathrm{L}$. J Ethnopharmacol 89: 131-137.

37. Rodanant $P$, Khetkam P, Suksamrarn A, Kuvatanasuchati J (2015) Coumarins and flavonoid from Murraya paniculata (L.) Jack: Antibacterial and antiinflammation activity. Pak J Pharm 28: 1947-1951.

38. Dong R, Yuan J, Wu S, Huang J, Xu X, et al. (2015) Anti-inflammation furanoditerpenoids from Caesalpinia minax Hance. Phytochem 117: 325-331.

39. Lian L, Li XB, Yuan JZ, Cheng L, Wu ZH, et al. (2015) Two new diterpenes from the seeds of Caesalpinia minax Hance. J Asian Nat Prod Res 17: 893-899.

40. Rubel R, Dalla Santa HS, Bonatto SJ, Bello S, Fernandes LC, et al. (2010) Medicinal mushroom Ganoderma lucidum (Leyss: Fr) Karst. triggers immunomodulatory effects and reduces nitric oxide synthesis in mice. J Med Food 13: 142-148.

41. de Souza MM, Madeira A, Berti C, Krogh R, Yunes RA, et al. (2000) Antinociceptive properties of the methanolic extract obtained from Ipomoea pes-caprae (L.) R. Br. J Ethnopharmacol 69: 85-90.

42. Ma KJ, Zhu ZZ, Yu CH, Zhang H, Liu J, et al. (2011) Analgesic, antiinflammatory, and antipyretic activities of the ethanol extract from Desmodium caudatum. Pharm Biol 49: 403-407.

43. Min KR, Hwang BY, Lim HS, Kang BS, Oh GJ, et al. (1999) (-)-Epiafzelechin cyclooxygenase- 1 inhibitor and anti-inflammatory agent from aerial parts of Celastrus orbiculatus. Planta Med 65: 460-462.

44. Khedr Al, Ibrahim SR, Mohamed GA, Ahmed HE, Ahmad AS, et al. (2016) New ursane triterpenoids from Ficus pandurata. Arch Pharm Res 39: 897-911.

45. Park HJ (2014) Immune stimulatory activity of BRP-4, an acidic polysaccharide from an edible plant, Basella rubra L. Asian Pac J Trop Med 7: 849-853.

46. Chang BY, Kim SB, Lee MK, Park H, Kim SY (2015) Improved chemotherapeutic activity by Morus alba fruits through immune response of toll-like receptor 4 . Int J Mol Sci 16: 24139-24158.

47. Khunakornvichaya A, Lekmeechai S, Pham PP, Himakoun W, Pitaksuteepong T, et al. (2016) Morus alba L. Stem Extract Attenuates Pain and Articular Cartilage Damage in the Anterior Cruciate Ligament Transection-Induced Rat Model of Osteoarthritis. Pharmacology 98: 209-216.

48. Yang S, Wang BL, Li Y (2014) Advances in the pharmacological study of Morus alba L. Yao Xue Xue Bao 49: 824-831.

49. Cho HY, Cho CW, Song YS (2005) Antioxidative and anti-inflammatory effects of Saururus chinensis methanol extract in RAW 264.7 macrophages. J Med Food 8: 190-197.

50. Kim RG, Shin KM, Kim YK, Jeong HJ, Ha J, et al. (2003) Inhibition of methano extract from the aerial parts of Saururus chinensis on lipopolysaccharideinduced nitric oxide and prostagladin E2 production from murine macrophage RAW 264.7 cells. Biol Pharm Bull 26: 481-486.

51. Li B, Lee YJ, Kim YC, Yoon JJ, Lee SM, et al. (2014) Sauchinone from Saururus chinensis protects vascular inflammation by heme oxygenase- 1 induction in 
Citation: Chen P, Zheng F, Zhang Y, Gao F, Chen Y, et al. (2016) Ethnobotanical Study of Medicinal Plants on Arthritis Used by Chaoshan in Guangdong, China. Med Chem (Los Angeles) 6: 715-723. doi: 10.4172/2161-0444.1000420

human umbilical vein endothelial cells. Phytomedicine 21: 101-108.

52. Meng X, Kim I, Jeong YJ, Cho YM, Kang SC (2016) Anti-inflammatory effects of Saururus chinensis aerial parts in murine macrophages via induction of heme oxygenase-1. Exp Biol Med 241: 396-408.

53. Geissberger P, Séquin URS (1991) Constituents of Bidens pilosa L.: do the components found so far explain the use of this plant in traditional medicine? Acta Trop 48: 251-261.

54. Horiuchi M, Wachi H, Seyama Y (2010) Effects of Bidens pilosa L. var. radiata Scherff on experimental gastric lesion. J Nat Med 64: 430-435.

55. Lu CL, Zhu YF, Hu MM, Wang DM, Xu XJ, et al. (2015) Optimization of astilbin extraction from the rhizome of Smilax glabra, and evaluation of its antiinflammatory effect and probable underlying mechanism in lipopolysaccharideinduced RAW264. 7 macrophages. Molecules 20: 625-644.

56. Patel NK, Pulipaka S, Dubey SP, Bhutani KK (2014) Pro-inflammatory cytokines and nitric oxide inhibitory constituents from Cassia occidentalis roots. Nat Prod Commun 9: 661-664.

57. Saleem TM, Azeem AK, Dilip C, Sankar C, Prasanth NV, et al. (2011) Antiinflammatory activity of the leaf extacts of Gendarussa vulgaris Nees. Asian Pac J Trop Med 1: 147-149

58. Chen Y, Tao S, Zeng F, Xie L, Shen Z (2015) Antinociceptive and antiinflammatory activities of Schefflera octophylla extracts. J Ethnopharmacol 171: $42-50$.

59. Yang BY, Guo R, Li T, Liu Y, Wang CF, et al. (2014) Five withanolides from the leaves of Datura metel $\mathrm{L}$. and their inhibitory effects on nitric oxide production. Molecules 19: 4548-4559.
60. Yang BY, Guo R, Li T, Wu JJ, Zhang J, et al. (2014) New anti-inflammatory withanolides from the leaves of Datura metel L. Steroids 87: 26-34.

61. Yang K, Tong L, Chen C, Zhang P, Pi H, et al. (2013) Therapeutic effects of extracts from Radix Toddaliae Asiaticae on collagen-induced arthritis in Balb/c mice. J Ethnopharmacol 146: 355-362.

62. Pereira RL, Ibrahim T, Lucchetti L, da Silva AJR, de Moraes VLG (1999) Immunosuppressive and anti-inflammatory effects of methanolic extract and the polyacetylene isolated from Bidens pilosa L. Immunopharmacol 43: 31-37.

63. Chih HW, Lin CC, Tang KS (1995) Anti-inflammatory activity of Taiwan folk medicine" ham-hong-chho" in rats. Am J Chin Med 23: 273-278.

64. Muthuraman A, Singh N (2011) Attenuating effect of Acorus calamus extract in chronic constriction injury induced neuropathic pain in rats: an evidence of antioxidative, anti-inflammatory, neuroprotective and calcium inhibitory effects. BMC Complement Altern Med 11: 24-37.

65. Chen L, Lan Z, Zhou Y, Li F, Zhang X, et al. (2011) Astilbin attenuates hyperuricemia and ameliorates nephropathy in fructose-induced hyperuricemic rats. Planta Med 77: 1769-1773.

66. Wang CP, Wang Y, Wang X, Zhang X, Ye JF, et al. (2011) Mulberroside a possesses potent uricosuric and nephroprotective effects in hyperuricemic mice. Planta Med 77: 786-794.

67. Yu Z, Fong WP, Cheng CH (2007) Morin (3, 5, 7, 2', 4'-pentahydroxyflavone) exhibits potent inhibitory actions on urate transport by the human urate anion transporter (hURAT1) expressed in human embryonic kidney cells. Drug Metab Dispos 35: 981-986. 